\title{
Synthesis and characterization of molecular semiconductors based on aromatic diamines with various electron acceptors
}

\author{
RAM ADHAR SINGH* and O SUNDAR RAO \\ Department of Chemistry, Faculty of Science, Banaras Hindu University, Varanasi 221005 , \\ India
}

MS received 25 January 1995; revised 8 June 1995

\begin{abstract}
The synthesis, spectral, electrical and magnetic properties of charge transfer materials of aromatic diamines namely benzidine (BEN) and $\rho$-toluidine (O.TOL) with various acceptors such as iodine, 2,3-dichloro-5,6-dicyano-p-benzoquinone (DDQ), tetracyanoquinodimethane (TCNQ), and $p$-chloranil ( $p$-CA) have been reported. These materials except $o$-toluidine-chloranil complex have been found to be typical molecular semiconductors on the basis of temperature dependence on their electrital conductivity. They have low electrical conductivities and two thermal activation energies indicating mixed conductivity mechanism in which both the electrons and holes are charge carriers, one dominating over the other at different temperatures. The observation of the metal like electrical conductivity i.e. the increase of electrical conductivity with decreasing temperature in the o-toluidinechloranil system is unique for this class of molecular electronic materials.
\end{abstract}

Keywords. Charge transfer materials; molecular semiconductors.

\section{Introduction}

Organic charge transfer solids offer a wide range of materials from insulators to superconductors (Gutman and Lyons 1967; Haddon et al 1984; Bryce 1991). These materials contain electron donors and electron acceptors in which electrons are exchanged between the two constituents. Most of the attention has been paid to TTF-TCNQ family of materials (Wheland and Gilson 1976). Aromatic diamines are another family of electron donors from which highly conducting and semiconducting complexes mainly with iodine as acceptor have been prepared e.g. the iodine complex of $p$-phenylenediamine has been studied for its electrical conductivity at various iodine concentrations having non-stoichiometrical compositions (Nishizaki and Kusakawa 1963). The electrical conductivity of the iodine complexes of various aromatic diamines such as 1,5-naphthylenediamine, benzidine, $o$-toluidine and $p, p^{\prime}$-diaminodiphenyl methane were studied for their d.c. conductivity at different temperatures. It has been found that resistivity decreases with increase in iodine content in the range of 0.75 to $1.50 \mathrm{I}_{2}$ /diamine mixed mole ratios. This observation has been explained on the basis of variable coordination number of aromatic diamineiodine complexes (Kusakawa and Nishizaki 1965). The electrical conductivities of solid $\mathrm{C}-\mathrm{T}$ complexes such as benzidine-iodine, phenothiazine-iodine, $p$ phenylenediamine-iodine, phenothiazine-SbCl${ }_{5}$ have been studied and the effect of ambient gases on conductivity was examined (Bhat and Rao 1969). Benzidine-iodine complex of various ratios has also been used as cathode material in the fabrication of solid state galvanic cells (Scrosati and Torroni 1973; Singh and Rao 1992). The non-ohmic conduction and electrical switching in benzidine-DDQ complex has been

\footnotetext{
* For correspondence
} 
studied at high pressures and found the switching behaviours at high electric fields $\left(3 \times 10^{3} \mathrm{~V} / \mathrm{cm}\right)$ (Ravindran and Subramanyam 1991). The effect of pressure on electrical conductivity of some diamine-chloranil complexes have been studied (Sakata et al 1974). It was found that the resistance initially decreased with increasing pressure, then increased and decreased again at higher pressures.

Electronic properties of some complexes of TCNQ with aromatic diamines were studied (Ohmasa et al 1968). These complexes showed a variety of interesting magnetic, electrical and optical properties. In search of an alternative to TTF family of conducting charge transfer materials, we have prepared and characterized many phenothiazine based materials (Singh et al 1990, 1991a-d, 1993). Continuing our effort in this direction, we are now presenting a detailed study on charge transfer materials based on some aromatic diamines such as benzidine (BEN) and $o$-toluidine (O.TOL) with various electron acceptors such as iodine, 2,3-dichloro-5,6-dicyano-p-benzoquinone (DDQ), p-chloranil ( $p . C A)$, tetracyanoquinodimethane (TCNQ). These systems were chosen with the objective of assessing their solid state properties which would help us in identifying materials for the fabrication of Langmuir-Blodgett films, because aromatic diamines could be easily substituted by long alkyl chains. Further these materials are suitable for the fabrication of organic batteries as reported earlier (Singh et al 1992, 1995).

The structural formulae of different electron donors and electron acceptors studied in the present work is given below:<smiles>Nc1ccc(-c2ccc(N)cc2)cc1</smiles>

(BEN)<smiles>N#CC1=C(C#N)C(=O)C(Cl)=C(Cl)C1=O</smiles>

(DDQ)<smiles>O=C1C(Cl)=C(Cl)C(=O)C(Cl)=C1Cl</smiles>

$\left(p-C_{A}\right)$<smiles>N#CC(C#N)=c1ccc(=C(C#N)C#N)cc1</smiles>

\section{Experimental}

\subsection{Materials and methods}

2,3-dichloro-5,6-dicyano-p-benzoquinone (DDQ), tetracyanoquinodimethane (TCNQ), 
p-chloranil ( $p . \mathrm{CA}$ ) of Aldrich, USA, benzidine (Stains, London), and $o$-toluidine $(\mathrm{CDH}, \mathrm{Delhi})$ were purified by recrystallization. The purities of these compounds were tested from their thin layer chromatography, melting point and infrared spectra which were in agreement with the literature data. The charge transfer complexes of benzidine and $o$-toluidine with iodine, 2,3-dichloro-5,6-dicyano-pbenzoquinone, tetracyanoquinodimethane and $p$-chloranil were generally prepared by mixing the appropriate amounts of components in the desired molar ratios in acetonitrile. o-Toluidine-chloranil complex was prepared by solid state method in which both the components were taken in a test tube and heated at approximately $300^{\circ} \mathrm{C}$ in a furnace since the complex was not precipitated from the solution.

\subsection{Measurements}

2.2a Electrical conductivity: Our attempts to grow single crystals by slow evaporation from very dilute solutions containing these complexes failed. So, the electrical measurements were done on compacted pellets. The samples were ground to a fine powder and pressed as pellets using the hydraulic press (Spectralab, India) at a pressure of $10 \mathrm{kbars}$. Platinum and graphite metals were used as contacts. The capacities of the same samples were measured by LCR meter (LCR 7 Vasavi, Hyderabad) and their dielectric constants calculated. The d.c. electrical conductivities at different temperatures were measured by using a Source Measure Unit (Keithley, USA, Model 236). The temperature of a mini-oven in which the pressed pellets were kept in a brass zig, was controlled and measured using a Temperature Indicator-Controller (Century Instruments, Chandigarh) with an accuracy of $\pm 0.5 \mathrm{C}$.

2.2b IR spectra and magnetic susceptibility: IR absorption spectra of all the complexes and components were recorded on JASCO FT-IR 5300 unit in $\mathrm{KBr}$ medium. Magnetic susceptibilities and respective magnetic moments were determined using a Cahn Faraday electrobalance.

\section{Results and discussion}

\subsection{IR spectral characterization}

The formation of complexes of BEN and O.TOL with iodine, DDQ, p. CA, TCNQ has been indicated by comparing the infrared spectra of individual components with their complexes (figures 1 and 2). The characteristic extra bands in the spectra of complex which are not present in its components indicate the formation of complex. Sharp $\mathrm{N}-\mathrm{H}$ peaks of pure donors have been replaced by broad bands indicating the existence of $\mathrm{N}^{+}-\mathrm{H}$ possibly due to the formation of cation radicals. The IR spectra of DDQ complexes of benzidine and $o$-toluidine show characteristic semiquinone peaks indicating the proton transfer from benzidine to DDQ apart from the electron transfer from the donor to the acceptor. Shifts in band position of $-\mathrm{C} \equiv \mathrm{N}$ and $-\mathrm{N}-\mathrm{H}$ groups indicate the formation of moderately strong complexes of benzidine and o-toluidine with DDQ and TCNQ. 


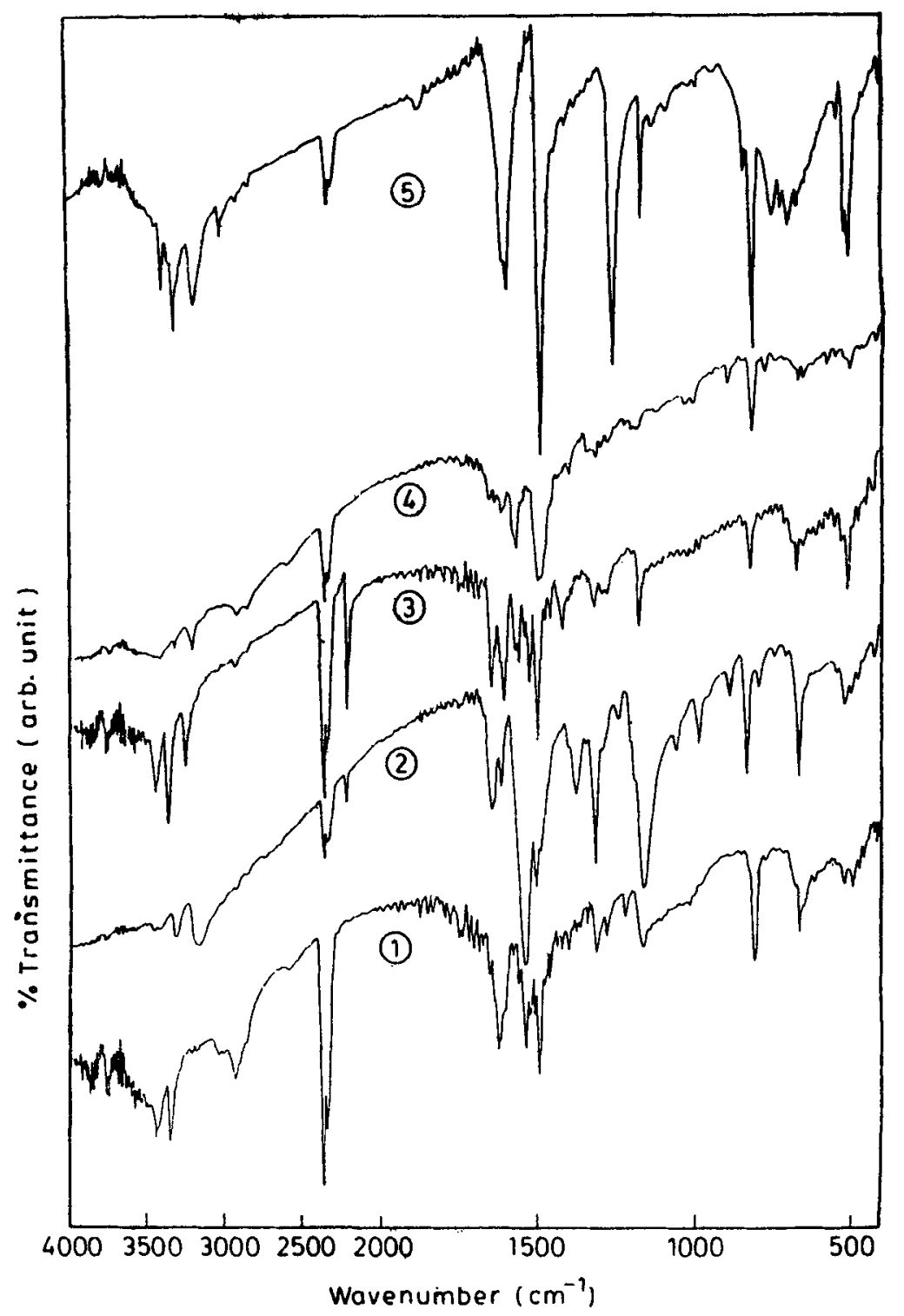

Figure 1. Infrared spectra of benzidine complexes of iodine(1), DDQ(2), TCNQ(3), p-CA(4) and $\operatorname{BEN}(5)$.

\subsection{Electrical and magnetic properties}

The dc conductivity of all the complexes at platinum contacts have been studied at room temperature by means of $I-V$ characteristics in which variable voltages were applied to the samples and corresponding currents measured.

The majority of the complexes have been found to show ohmic behaviour when they were subjected to forward and reverse bias. The electrical and magnetic moment data of various complexes of BEN and O.TOL are presented in table 1 . The electrical 


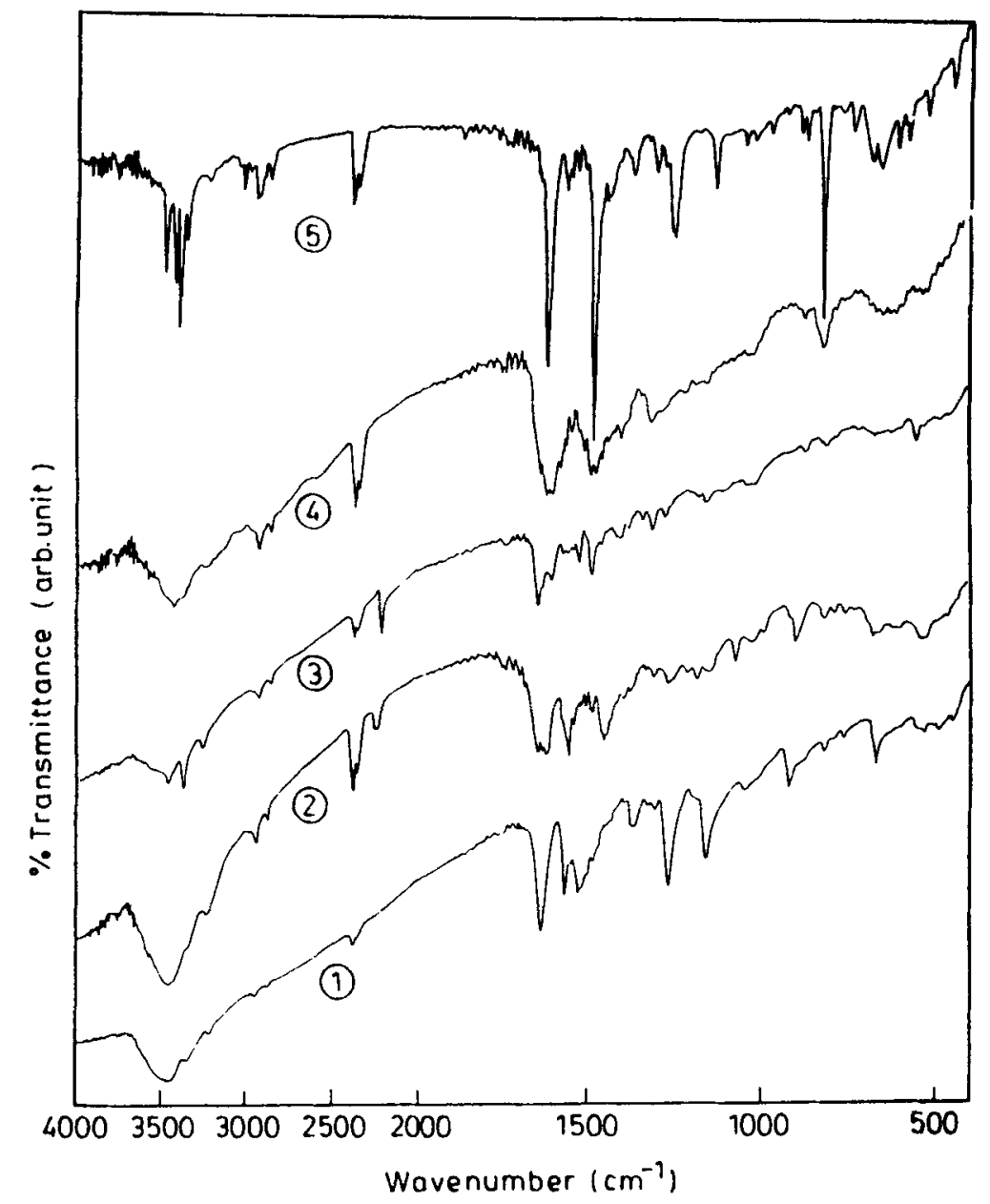

Figure 2. Infrared spectra of a-toluidine complexes of iodine(1), DDQ(2), TCNQ(3), p.CA(4) and O.TOL(5).

conductivity and dielectric constants of DDQ and p.CA complexes are found to be higher than the other complexes. For example, BEN-p.CA complex has conductivity $1.8 \times 10^{-6} \mathrm{~S} \mathrm{~cm}^{-1}$ and dielectric constant value as 86.5 . However, the values of electrical conductivities of benzidine-iodine complex obtained in this investigation are 3-4 orders of magnitude lower than the values reported (Kusakawa and Nishizaki 1965). This may possibly be due to solvent dependent grain sizes in the powder which affect the inter-particle contacts in the pressed pellets. Also, the metallic contacts used to connect the pellets with SMU may inject or block charge carriers. Platinum, a noble metal with a high positive electrode potential, was used in our study to avoid reaction with pellet. Graphite has also been tested as a contact which gives more or less similar results. The high resistivity of these samples may be due to the existence of grain boundaries and space charges at the interface of platinum foil and the sample because of the uneven surfaces of pellets and existence of surface states (Gutman and Lyons 1967). These values are fairly stable and reproducible even after a period of 5 months. 
The temperature dependence of conductivity was studied by applying constant voltage to the sample and measuring the corresponding current at variable temperatures and they are presented in figures 3 and 4 . These experiments were performed for at least two times in order to ensure their reproducibility. In all the systems, except in $o$-toluidine-chloranil complex, the electrical conductivity increased with temperature indicating a typical molecular semiconducting behaviour. Single activation energies were found with all the systems except in BEN-iodine, O.TOL-iodine and O.TOL-DDQ in

Table 1. Electrical and magnetic properties of charge transfer complexes of benzidine(BEN) and $o$-toluidine(O.TOL) with iodine, DDQ, p.CA and TCNQ at room temperature.

\begin{tabular}{lllllll}
\hline & $\begin{array}{c}\text { Specific } \\
\text { conductance } \\
\left.(\mathrm{S} \mathrm{cm})^{-1}\right)\end{array}$ & $\begin{array}{c}\text { Energy of } \\
\text { activitation } \\
\text { Ea }\end{array}$ & $\begin{array}{c}\mathrm{Ea}_{2} \\
(\mathrm{eV})\end{array}$ & $\begin{array}{c}\text { Capacitance } \\
(\mathrm{eV})\end{array}$ & $\begin{array}{c}\text { Dielectric } \\
\text { constant } \\
\left(\mathrm{K}=\mathrm{C}_{\mathbf{C}} \mathrm{C}_{0}\right)\end{array}$ & $\begin{array}{c}\text { Magnetic } \\
\text { moment } \\
(\mathrm{BM})\end{array}$ \\
\hline BEN-iodine & $3.5 \times 10^{-8}$ & 0.52 & 0.38 & 16.4 & 21.3 & 0 \\
BEN-DDQ & $2.03 \times 10^{-5}$ & 0.19 & - & 16.5 & 15.1 & 0.92 \\
BEN-CA & $1.8 \times 10^{-6}$ & 0.13 & - & 64.0 & 86.5 & 0 \\
BEN-TCNQ & $3.2 \times 10^{-9}$ & 0.06 & - & 29.4 & 16.2 & 0 \\
O.TOL-iodine & $5.9 \times 10^{-8}$ & 0.69 & 0.28 & 10.9 & 4.8 & 0 \\
O.TOL-DDQ & $1.31 \times 10^{-7}$ & 0.39 & 0.46 & 14.5 & 8.0 & 0 \\
O.TOL-CA & $2.3 \times 10^{-5}$ & 0.56 & - & 14.1 & 19.1 & 0 \\
O.TOL-TCNQ & $1.88 \times 10^{-7}$ & 0.86 & - & 17.0 & 32.1 & 1.94
\end{tabular}

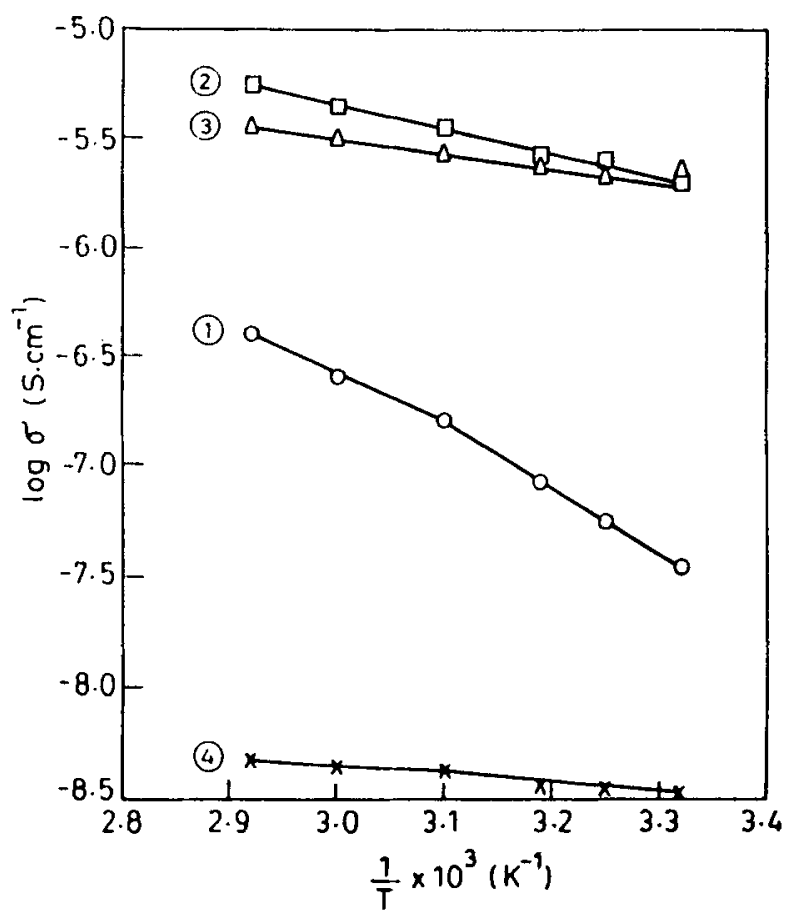

Figure 3. The plot of log of conductivity of benzidine-iodine(1), BEN-DDQ(2), BENTCNQ(3) and BEN-p.CA(4). 


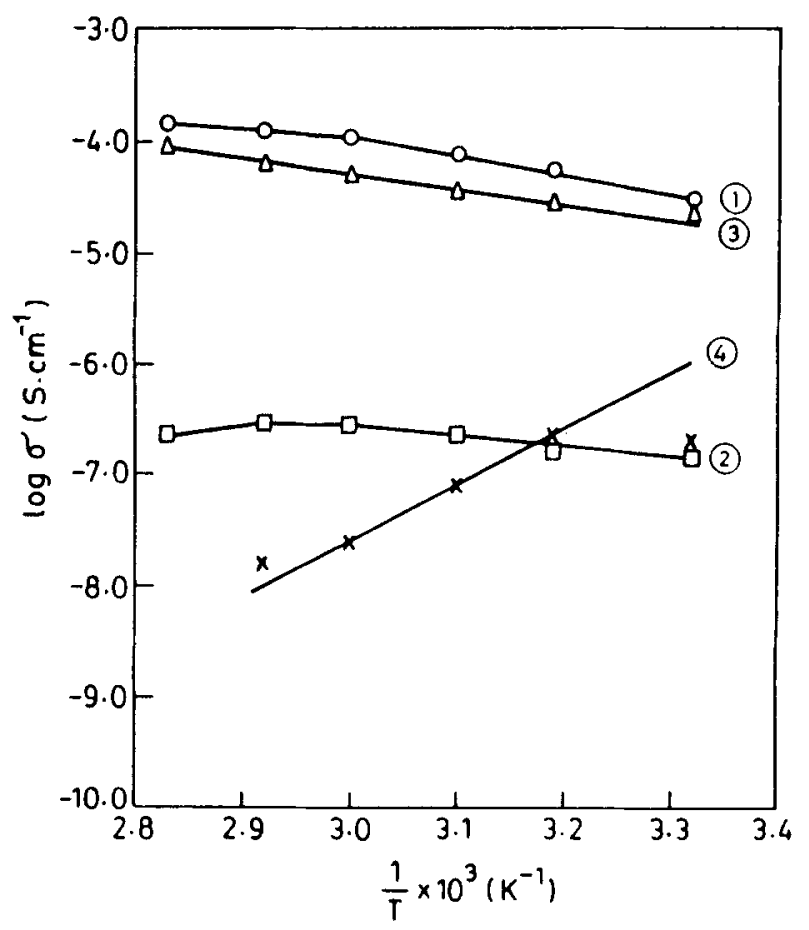

Figure 4. The plot of log of conductivity of $o$-toluidine-iodine(1), O.TOL-DDQ(2), O.TOLTCNQ(3) and O.TOL-p.CA(4).

which two activation energies were obtained from their nonlinear curves. The existence of two energies of activation may be due to the mixed conductivity, i.e. both by electrons and holes, one being the major carrier at around room temperature and the other at higher temperatures as already proposed (Bandrauk et al 1985). The existence of some ionic (proton) contribution to the electrical conduction, apart from electronic ones in BEN-I ${ }_{2}$ and O.TOL-I ${ }_{2}$ systems, was found and it is being investigated further (Singh et al 1995). Capacities were found to increase with rise in temperature in majority of these systems, which is a characteristic of semiconductors. Dielectric constants were calculated for all the complexes and the highest value of 86.5 for BEN-p.CA complex was found (table 1).

Unusual metallic behaviour of O.TOL-p.CA system in the temperature range 303$370 \mathrm{~K}$ for weak complexes seems to be similar to diamine-TCNQ(1:2) complex which showed temperature independent of electrical and magnetic behaviour (Ohmasa et al 1968).

The magnetic measurements for the majority of the complexes showed no change in the weight of sample in presence of $10 \mathrm{~K}$ Gauss magnetic field indicating that they were diamagnetic. But in the case of BEN-DDQ and O.TOL-TCNQ complexes, magnetic moment values of 0.92 and 1.94 Bohr magnetons were obtained respectively, which may be due to the presence of unpaired spins.

It can be concluded that benzidine and $o$-toluidine forms complexes with iodine, DDQ, p.CA and TCNQ showing typical molecular semiconduction with one/two activation energies. The electrical conductivities of these complexes have been found to vary in following order: BEN-DDQ $>$ BEN-CA $>$ BEN-iodine $>$ BEN-TCNQ and 
O.TOL-CA > O.TOL-TCNQ > O.TOL-DDQ > O.TOL-iodine. IR spectral data indicate the complex formation between components and the magnetic studies reveal that majority of them are diamagnetic except BEN-DDQ and O.TOL-TCNQ complexes which contain unpaired spins.

\section{Acknowledgement}

We are thankful to the Council of Scientific and Industrial Research, New Delhi for financial support.

\section{References}

Bandrauk A D, Ishi K D, Truong K D, Aubin M and Hanson A W 1985 J. Phys. Chem. 891478 Bhat S N and Rao C N R 1969 Can. J. Chem. 473899

Bryce M R 1991 Chem. Soc. Rev. 20355

Gutman F and Lyons L E 1967 Organic semiconductors (New York: John Wiley \& Sons Inc.)

Haddon R C, Kaplan M L and Wudl F 1984 Encyclopedia of semiconductor technology (ed.) M Grayson (New York: Wiley Interscience)

Kusakawa $H$ and Nishizaki S 1965 Bull. Chem. Soc. Jpn 38313

Nishizaki S and Kusakawa H 1963 Bull. Chem. Soc. Jpn 361681

Ohmasa M, Kinoshita M, Sano M and Akamatu H 1968 Bull. Chem. Soc. Jpn 411998

Ravindran T and Subramanyam S V 1991 Bull. Mater. Sci. 141205

Sakata T, Akifumi O, Hiroshi T and Naoto K 1974 J. Am. Chem. Soc. 963365

Scrosati B and Torrini M 1973 Electrochim. Acta 18225

Singh R A and Rao O S 1992 Bull. Electrochem. 8468

Singh R A, Singh R and Narayan R 1990 Bull. Electrochem. 6519

Singh R A, Singh R and Verma S M 1991a Cryst. Res. Tech. 26201

Singh R A, Singh R and Verma S M 1991 b Cryst. Res. Tech. 26339

Singh R A, Singh R and Verma S M 1991c Indian J. Tech. 29241

Singh R A, Singh R and Verma S M 1991d Bull. Chem. Soc. Jpn 641938

Singh R A, Singh R, Rao O S and Verma S M 1993 Mol. Cryst. Liq. Cryst. 237419

Singh R A, Rao O S and Singh V K 1995 Indian J. Mater. Sci. Engg. (accepted)

Wheland R C and Gilson J L 1976 J. Am. Chem. Soc. 983916 